F.J. Gilchrist*, H. Alton", M-A Brundler ${ }^{\natural}$, L. Edwards ${ }^{+}$, A. Plunkett ${ }^{+}$and S. Rao*

Depts of *Paediatric Respiratory Medicine, "Radiology, "Pathology, and 'Paediatric Intensive Care, Birmingham Children's Hospital, Birmingham, UK.

Correspondence: S. Rao, Dept of Paediatric Respiratory Medicine, Birmingham Children's Hospital, Steelhouse Lane, Birmingham, B4 6NH, UK. E-mail: satish.rao@bch.nhs.uk

Statement of Interest: S. Rao has received educational grants from Novartis (UK) and GlaxoSmithKline (UK) for attending conferences and delivering lectures as part of an educational programme organised by the two companies.

Provenance: Submitted article, peer reviewed.

\section{REFERENCES}

1 Bruce DM, Heys SD, Eremin O. Lymphangitis carcinomatosa: a literature review. J $R$ Coll Surg Edinb 1996; 41: 7-13.
2 Vanclaire J, Bodart E, Schlesser P, et al. Lymphangite carcinomateuse pulmonaire et adénocarcinome rénal [Pulmonary carcinomatous lymphangitis and renal adenocarcinoma]. Arch Fr Pediatr 1990; 47: 735-736.

3 Dennstedt FE, Greenberg SD, Kim HS, et al. Pulmonary lymphangitic carcinomatosis from occult stomach carcinoma in young adults: an unusual cause of dyspnea. Chest 1983; 84: 787-788.

4 Thomas A, Lenox R. Pulmonary lymphangitic carcinomatosis as a primary manifestation of colon cancer in a young adult. CMAJ 2008; 179: 338-340.

5 Section VI: Pulmonary neoplasms. In: Fraser RS, Muller NL, Colman NC, et al., eds. Fraser and Pare's Diagnosis of Diseases of the Chest. Philadelphia, Elsevier Health Sciences, 1999; pp. 1390-1397.

6 Masson RG, Krikorian J, Lukl P, et al. Pulmonary microvascular cytology in the diagnosis of lymphangitic carcinomatosis. $N$ Engl J Med 1989; 321: 71-76.

7 Defranchi S, Bertolotti AM, Vigliano CA, et al. Open lung biopsy for diffuse disease in patients with and without previously transplanted solid organs. Ann Thorac Surg 2010; 90: 965-971.

8 Kikuchi N, Shiozawa T, Ishii Y, et al. A patient with pulmonary lymphangitic carcinomatosis successfully treated with TS-1 and cisplatin. Intern Med 2007; 46: 491-494.

\title{
Pulmonary nocardiosis in immunocompetent patients: can COPD be the only risk factor?
}

\section{To the Editor:}

Pulmonary nocardiosis is a rare disorder that mainly affects immunocompromised patients. Several risk factors have been identified, such as corticosteroid therapy, chronic obstructive pulmonary disease (COPD), cystic fibrosis and bronchiectasis. Diagnosis of nocardiosis is difficult as bacteriological culture can be problematic. However, if observations are atypical, clinicians should consider Nocardia in immunocompetent patients, particularly when the patient is also suffering from COPD. Herein, we ask whether COPD alone is a risk factor for pulmonary nocardiosis.

We report the case of a 71-yr-old female smoker suffering from COPD who had not received inhaled or oral (bolus or long-term) corticosteroid therapy. She had suffered from bronchiectasis after pulmonary tuberculosis 60 yrs previously. She presented to the hospital with an exacerbated chronic cough that had lasted several months and chronic fever, with no weight loss. Prior to admission, amoxicillin followed by spiramycin therapy was not effective. Laboratory evaluation showed hyperleukocytosis $\left(14,130\right.$ cells per $\mathrm{mm}^{3}$, with 10,753 polynuclear neutrophils per $\mathrm{mm}^{3}$ ) and C-reactive protein $75 \mathrm{mg} \cdot \mathrm{L}^{-1}$ (normal range $<5 \mathrm{mg} \cdot \mathrm{L}^{-1}$ ), with no renal failure or hepatic biological abnormalities. Her gamma globulin level was normal $\left(11.7 \mathrm{~g} \cdot \mathrm{L}^{-1}\right)$. Aspergillosis serology and the tuberculin intradermal reaction were negative; sputum examination was sterile, without Mycobacterium or Aspergillus. Chest radiography showed linear opacity of the right upper lobe with bronchiectasis and excavated opacity in the right upper lobe. A computed tomography scan of the chest revealed bronchiectasis with a thickened lining and cystic dilatation of the right upper lobe, as well as diffuse centrolobular micronodules in all lobes of the right lung (fig. 1).

On bronchoscopy the bronchial mucous membrane was normal but had a suppurating secretion. Microbiological evaluation of bronchoscopic samples did not reveal any Mycobacterium or Aspergillus species, but did reveal a Nocardia species identified as Nocardia cyriacigeorgica from 16S rRNA gene sequencing in the French Nocardiosis Observatory (Lyon, France). Antibiotic susceptibility was tested. The isolate was sensitive to amoxicillin-clavulanic acid (minimum inhibitory concentration (MIC): $1.2 \mu \mathrm{g} \cdot \mathrm{mL}^{-1}$ ), cefotaxime (MIC: $0.04 \mu \mathrm{g} \cdot \mathrm{mL}^{-1}$ ), amikacin (MIC: $0.01 \mu \mathrm{g} \cdot \mathrm{mL}^{-1}$ ) and trimethoprim-sulfamethoxazole (MIC: $\left.0.002 \mu \mathrm{g} \cdot \mathrm{mL}^{-1}\right)$.

An intravenous antimicrobial therapy of trimethoprimsulfamethoxazole (cotrimoxazole) and amikacin was administered for 15 days followed by oral only trimethoprimsulfamethoxazole for 6 months. The patient's clinical condition gradually improved over the course of her stay in the hospital. At 4 months after discharge from the hospital, follow-up radiological investigations showed a favourable outcome. Alveolar condensation and centrolobular micronodules were resolved, but cystic bronchiectasis persisted within the fine lining (fig. 1). The clinical, biological and radiological outcomes were still favourable, without a relapse 12 months after the end of antimicrobial therapy.

A pulmonary infection caused by Aspergillus can cause the same radiological abnormalities in patients suffering from COPD as 

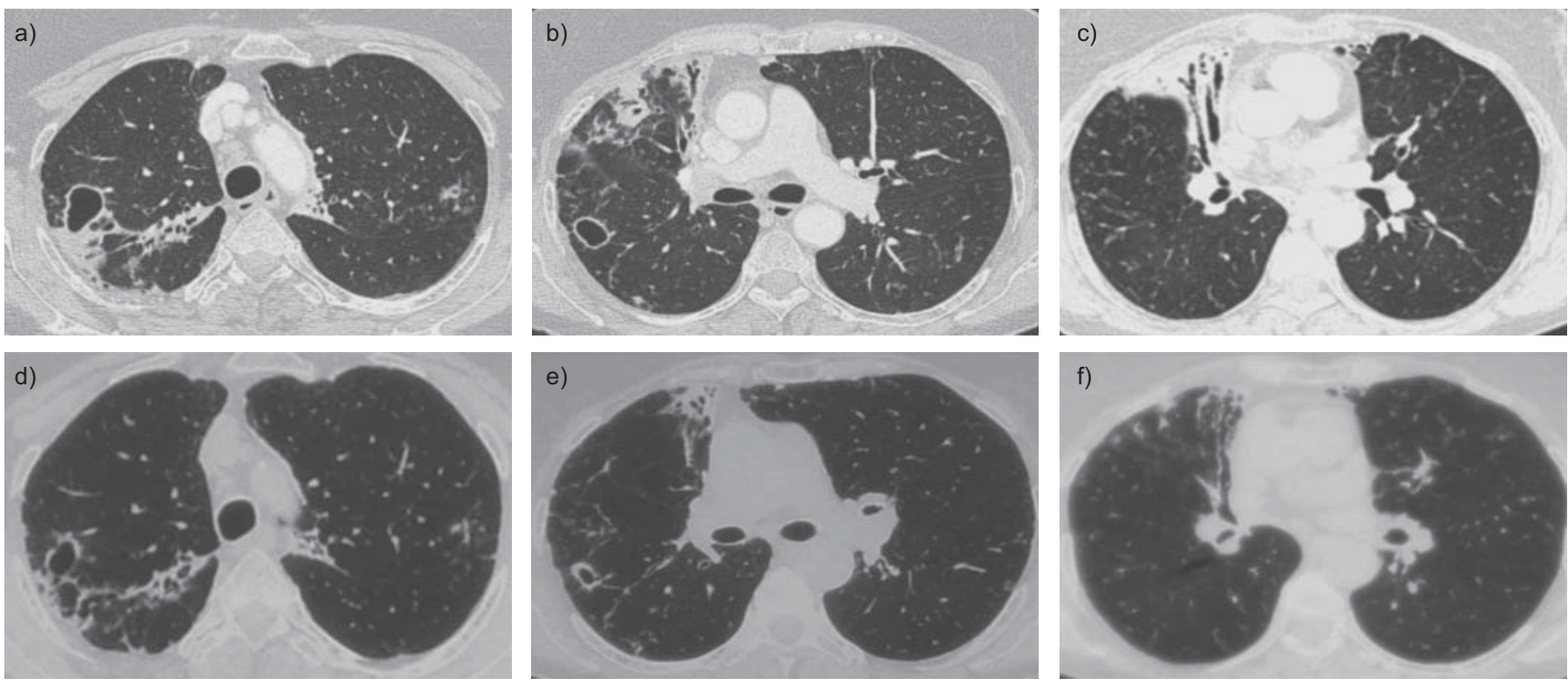

FIGURE 1. Chest computed tomography scans at a-c) the beginning of antimicrobial therapy (August 2009) and d-f) after 4 months of antimicrobial therapy (December 2009) showing regression of opacities and cystic bronchiectasis.

those seen in N. cyriacigeorgica infections. However, galactomannan antigenaemia testing and Aspergillus serologies were negative in our patient. No Aspergillus infection was found in the bronchial aspiration or the bronchoalveolar lavage analysis.

A patient with COPD may have increased susceptibility to fungal infection for several reasons, such as a change in bronchial architecture, the use of steroids, frequent hospitalisation or antibiotic treatments, comorbidity factors (alcoholism and diabetes, etc.), and these reasons may be linked with Nocardia colonisation or infection in patients suffering from COPD [1].

Nocardia is a ubiquitous aerobic Gram-positive bacteria from the order Actinomycetales [2-4]. Pulmonary nocardiosis is rare, but can be acute, subacute or chronic. The clinical symptoms are not specific (fever, cough and dyspnoea), and no biological or radiological sign is specific. Diagnosis is extremely difficult because nocardiosis is not well known by clinicians and the culture of Nocardia is not easy (it is important to inform biologists that Nocardia should be checked for) [2-4]. Dissemination of the bacteria is also possible and worsens the prognosis. Mortality related to nocardiosis is $\sim 15 \%[2,3]$.

The use of immunosuppressant agents (e.g. due to solid organ transplantation and haematopoietic stem cell transplantation) and corticosteroid therapies are the main risk factors for infection [3]. However, Nocardia can also affect non-immunocompromised hosts, possibly because of local impairment of bronchial defences and structural modifications to the bronchial architecture [1]. This can especially occur in the presence of COPD and bronchiectasis, which have both been reported as important risk factors for respiratory colonisation by Nocardia [3, 4]. Bacterial colonisation of the bronchus alters ciliary motility and causes epithelial damage, which can facilitate the presence of Nocardia [2]. Our patient had suffered from bronchiectasis after pulmonary tuberculosis.

Genetic susceptibility could explain why this infection occurs in immunocompetent patients. Not all patients infected with a pathogen develop clinical disease, and human genes play an important role in determining susceptibility or resistance. A complex interplay between environmental and human factors determines immunity to infection and the resulting clinical outcome of an infection. Most individuals retain a natural vulnerability to infectious diseases, reflecting the great diversity of potential inborn immunity errors. Studies that have compared the concordance rate of infectious diseases between monozygotic and dizygotic twins have implicated the host's genetic background in disease susceptibility. More common infectious diseases, caused by virulent microorganisms, may also be a result of Mendelian disorders [5, 6].

$N$. cyriacigeorgica is the new name for $N$. asteroides type IV [4]. This species was described in 2005 [6], but has been rarely reported in human infections [7].

Our patient was not immunocompromised (had not received corticosteroid therapy) and suffered from mild COPD. The role of COPD in infection risk is not fully known [2]. We found seven patients with COPD who also suffered from an N. cyriacigeorgica pulmonary infection in the literature (table 1) [8-11].

The data from these papers shows that: 1) sex ratio was slightly unfavourable to females $(1.25) ; 2)$ six $(85.7 \%)$ out of seven patients were aged $>70 \mathrm{yrs}$; 3) $100 \%$ of patients suffered from pulmonary nocardiosis, which was pulmonary only in $71.4 \%$ (five out of seven), had pleural involvement in 14.3\% (one out of seven) and involved endocarditis in $14.3 \%$ (one out of seven) of cases; 4$)$ five $(71.4 \%)$ out of seven patients had received corticosteroid therapy and one of these received it as an inhaled therapy; and 5) four (57.2\%) out of seven patients were treated with trimethoprim-sulfamethoxazole and one $(14.3 \%)$ had received amoxicillin and clavulanic acid.

Based on these published data, the mortality rate for pulmonary nocardiosis in patients suffering from COPD was 33\% (two out of six). 


\begin{tabular}{|c|c|c|c|c|c|c|}
\hline TABLE 1 & $\begin{array}{l}\text { ry of rep } \\
\text { ary disec }\end{array}$ & d cas & f Nocardia cyriacige & a infection in $\mathrm{p}$ & fering from & structive \\
\hline First author [ref.] & Age yrs & Sex & Corticosteroid therapy & Involvement & Treatment & Outcome \\
\hline \multirow[t]{4}{*}{ Casanova [6] } & 74 & Male & Oral steroid & Lung & Sulfamide & Survived \\
\hline & 44 & Female & Oral steroid & Lung & Cotrimoxazole & Survived \\
\hline & 71 & Female & Oral steroid & Lung & Cotrimoxazole & Died \\
\hline & 79 & Male & None & Lung & Co-amoxiclav & Survived \\
\hline MaRAKI [8] & 75 & Female & Oral and inhaled steroid & Lung/pleura & Cotrimoxazole & Died \\
\hline CARGILl [9] & 85 & Female & Oral steroid & Lung/endocarditis & Not available & Not available \\
\hline
\end{tabular}

These results should be interpreted cautiously because the number of patients is very small; nevertheless, there is a trend towards COPD being a risk factor for nocardiosis when it is associated with oral corticosteroid therapy, and infection is rare when there is no corticosteroid therapy. Our patient had never been prescribed oral or inhaled corticosteroid therapy, and no bacterial colonisation was found. The previously described pathophysiology of nocardiosis cannot be confirmed.

Antibiotic susceptibility of N. cyriacigeorgica showed the usual in vitro effectiveness of cotrimoxazole, imipenem, amikacin and linezolid, but also minocycline [3].

The duration of the antimicrobial therapies reported was not always clear. Nocardiosis should be treated for 6-12 months $[3,10]$. However, the duration of therapy also depends on the immunosuppressive agents and the underlying condition (e.g. COPD, HIV infection and solid organ transplantation).

In conclusion, this case report describes a rare and new aetiology of pulmonary infection caused by N. cyriacigeorgica. This case is atypical because the patient was not immunocompromised, suffered from mild COPD and had not received corticosteroid therapy. Because the diagnosis of nocardiosis can be difficult it is important to consider the possibility of Nocardia infection.

\section{F. Rivière*, M. Billhot*, C. Soler", F. Vaylet* and J. Margery*} *Respiratory Dept, and "Microbiology Dept, Percy Military Hospital, Clamart, France.

Correspondence: F. Rivière, Respiratory Dept, Percy Military Hospital, 101 avenue Henri Barbusse, 92140 Clamart, France. E-mail: frdriviere@orange.fr

Statement of Interest: None declared.
Provenance: Submitted article, peer reviewed.

\section{REFERENCES}

1 Ader F, Nseir S, Le Berre R, et al. Invasive pulmonary aspergillosis in chronic obstructive pulmonary disease: an emerging fungal pathogen. Clin Microbiol Infect 2005; 11: 427-429.

2 Martinez Tomas R, Menendez Villanueva R, Reyes Calzada S, et al. Pulmonary nocardiosis: risk factors and outcomes. Respirology 2007; 12: 394-400.

3 Minero MV, Marin M, Cercenado E, et al. Nocardiosis as the turn of the century. Medicine 2009; 88: 250-261.

4 Rodriguez-Nava V, Zoropoguy A, Laurent F, et al. La nocardiose, une maladie en expansion [Nocardiosis, an expanding disease]. Antibiotiques 2008; 10: 115-127.

5 Picard C, Casanova JL, Abel L. Mendelian traits that confer predisposition or resistance to specific infections in humans. Curr Opin Immunol 2006; 18: 383-390.

6 Casanova JL, Abel L. Inborn errors of immunity to infection: the rule rather than the exception. J Exp Med 2005; 202: 197-201.

7 Barnaud G, Deschamps C, Manceron V, et al. Brain abscess caused by Nocardia cyriacigeorgica in a patient with human immunodeficiency virus infection. J Clin Microbiol 2005; 43: 4895-4897.

8 Maraki S, Panagiotaki E, Tsopanidis D, et al. Nocardia cyriacigeorgica pleural empyema in an immunocompromised patient. Diagn Microbiol Infect Dis 2006; 56: 333-335.

9 Cargill JS, Boyd GJ, Weightman NC. Nocardia cyriacigeorgica: a case of endocarditis with disseminated soft-tissue infection. $J$ Med Microbiol 2010; 59: 224-230.

10 Munoz J, Mirelis B, Aragon LM, et al. Clinical and microbiological features of nocardiosis 1997-2003. I Med Microbiol 2007; 56: 545-550.

11 Akcaglar S, Yilmaz E, Heper Y, et al. Nocardia cyriacigeorgica: pulmonary infection in a patient with Basedow-Graves disease and a short review of reported cases. Int J Infect Dis 2008; 12: 335-338. 